\title{
Magnetic helicity accumulation and tilt angle evolution of newly emerging active regions
}

\author{
S. Yang ${ }^{1,2}$, H. Zhang ${ }^{1}$, and J. Büchner ${ }^{2}$ \\ 1 National Astronomical Observatories, Chinese Academy of Sciences, 100012 Beijing, PR China \\ e-mail: yangshb@bao.ac.cn \\ 2 Max-Planck Institute for Solar System Research, 37191 Katlenburg-Lindau, Germany
}

Received 23 April 2008 / Accepted 23 April 2009

ABSTRACT

\begin{abstract}
Context. It has been known for years that there is a general dominance of negative (positive) helicity of active regions (ARs) in the northern (southern) solar hemisphere. For a better understanding of the role of helicity in the evolution of active regions, it is necessary to know more about the accumulation of helicity during the emergence of active regions. In particular, different conclusions were drawn in the past about the relationship between the accumulated helicity and the writhe of active regions.

Aims. We investigate the accumulation of helicity in newly emerging simple bipolar solar active regions. We also investigate the relation between the accumulated helicity and writhe.

Methods. We obtain helicity accumulation by applying Fast Fourier Transforms (FFT) and local correlation tracking (LCT) to MDI data. We deduce the writhe of the active regions according to the evolution of the tilt angle between the connecting line of the weighting centers of opposite polarities in the ARs.

Results. It is found that the accumulated helicity is proportional to the exponent of magnetic flux $\left(|H| \propto \Phi^{1.85}\right)$ in the 58 selected newly emerged simple ARs. 74\% of ARs have a negative (positive) helicity when the above defined tilt angle rotates clockwise (counterclockwise). This means that the accumulated helicity and writhe have the same sign for most of the investigated ARs according to the tilt angle evolution of ARs. We also found that 56\% (57.6\%) of these ARs in the northern (southern) photosphere provide negative (positive) helicity to the corona in the course of the emergence of magnetic flux.
\end{abstract}

Key words. Sun: magnetic fields - Sun: activity

\section{Introduction}

Magnetic helicity is a key geometrical parameter to describe the structure evolution of the solar coronal magnetic field (cf. Berger 1999). Magnetic helicity in a volume $V$ is given by

$H_{\mathrm{M}}=\int_{V} \boldsymbol{A} \cdot \boldsymbol{B} \mathrm{d} V$

where $\boldsymbol{A}$ is the vector potential of magnetic field $\boldsymbol{B}$ in this volume. Magnetic helicity is conserved in ideal plasma (Wotjer 1958). It still conserves in ideal magneto hydrodynamics in the course of magnetic reconnection as long as the overall magnetic Reynolds number is large enough (Berger \& Field 1984). The corona in general satisfies the condition of ideal magnetohydrodynamics. Hence, the magnetic helicity in the solar atmosphere originates from below the photosphere. The accumulation of magnetic helicity in the solar atmosphere is associated with the increase of free energy which is released in the course of solar activity such as solar flares (Zhang 2006), filament eruptions (Chae 2001a), and coronal mass ejections (CMEs) (Zhang et al. 2006). It reflects the action of the solar dynamo (Seehafer et al. 2003; Kuzayan et al. 2003).

The vector potential $\boldsymbol{A}$ in formula (1) is not gauge-invariant. It cannot be determined directly from observations. So we need other methods to investigate the helicity properties in the solar atmosphere. Recently, many authors have used vector magnetographs to calculate the current helicity $h_{\mathrm{c}}=\mu_{0} B_{z} J_{z}$
(Abramenko et al. 1996; Bao \& Zhang 1998) or the force-free field parameter $\alpha_{\text {best }}$ (Pevtsov et al. 1995; Tian et al. 2001) of active regions that represent the helicity of the magnetic flux tubes. The shapes of sigmoid coronal loops were also studied (Pevtsov et al. 1995; Canfield \& Pevtsov 1999; Pevtsov et al. 2001). All these investigations revealed a dominance of negative (positive) helicity in the northern (southern) hemisphere, called the "Hemisphere Helicity Rule".

Berger \& Field (1984) argued that the relative magnetic helicity will also have a specific value in case of the open boundary conditions. A general expression for relative magnetic helicity is given by (Finn \& Antonsen 1985)

$H_{\mathrm{R}}=\int_{V}\left(\boldsymbol{A}+\boldsymbol{A}_{\mathrm{p}}\right) \cdot(\boldsymbol{B}-\boldsymbol{P}) \mathrm{d} V$

where $\boldsymbol{A}$ and $\boldsymbol{B}$ are the actual magnetic vector potential and magnetic field in the considered volume. $\boldsymbol{P}$ is the potential field and $\boldsymbol{A}_{\mathrm{p}}$ is the corresponding vector potential. $\boldsymbol{P}$ satisfies the following conditions:

$\nabla \times \boldsymbol{P}=0$

$\left.\boldsymbol{P} \cdot \widehat{n}\right|_{S}=\left.\boldsymbol{B} \cdot \widehat{n}\right|_{S}$.

The magnetic helicity flux across the open boundary in the ideal magnetohydrodynamics is given by (cf. Berger 1999)

$\frac{\mathrm{d} H_{\mathrm{R}}}{\mathrm{d} t}=-2 \int_{V}\left(\left(\boldsymbol{A}_{\mathrm{p}} \cdot \boldsymbol{V}\right) \boldsymbol{B}-\left(\boldsymbol{A}_{\mathrm{p}} \cdot \boldsymbol{B}\right) \boldsymbol{V}\right) \cdot \mathrm{d} \boldsymbol{S}$. 
The first term of expression (5) represents the effect of shearing motion of the boundary. The second term represents the bulk transport of the helical field across the boundary. Chae (2001a) utilized the local correlation tracking (LCT) and Fast Fourier Transform (FFT) methods to obtain the horizontal velocity field $\boldsymbol{V}$ and vector potential $\boldsymbol{A}_{\mathrm{p}}$ to calculate the accumulated helicity caused by the first term in formula (5). Demoulin \& Berger (2003) pointed out that the helicity given by Chae (2001) includes not only the emergence term but also the shear term if the photosphere is considered as an ideal plasma. Expression (5) becomes

$$
\frac{\mathrm{d} H_{\mathrm{R}}}{\mathrm{d} t}=-2 \int_{V}\left(\boldsymbol{A}_{\mathrm{p}} \cdot \boldsymbol{U}\right) \boldsymbol{B} \cdot \mathrm{d} \boldsymbol{S}
$$

where $\boldsymbol{U}$ is the horizontal velocity given by the LCT method.

The magnetic helicity of a solar flux tube can be decomposed into the twist around the flux tube axis and the writhe of the helical flux tube axis. Observations show that the flux tubes of most active regions exhibit significant magnetic writhe and twist at the photospheric level (e.g. Canfield \& Pevtsov 1998). Active regions are usually thought to be formed by magnetic flux tubes emerging from the core-convection zone interface (e.g. Gilman \& Charbonneau 1999) and a twist is needed to prevent the flux tube from being destroyed by the hydrodynamic vortex behind it during its emergence in the convective zone (Fan et al. 1998). When the flux rises from the convection zone, there are two main sources adding writhe to the flux tube axis. The first one is an internal instability such as kink instability (Leka et al. 1996; Linton et al. 1998; Fan et al. 1999). A kink instability causes the writhe of the tube axis to have the same sense as that of the twist within the tube due to conservation of helicity. The second source is the Coriolis force (cf. Fisher et al. 2000). As a flux tube in the northern (southern) hemisphere emerges through the convective zone, the Coriolis force will deform its main axis introducing positive (negative) writhe. An opposite but equal twist will be added to the flux tube because of helicity conservation. This effect also follows Joy's law in which the leading polarities are closer to the equator than the trailing polarities (Hale 1919). Hence, the relation between the twist and writhe in the active regions is important in analyzing effect of different sources and the origin of helicity. Based on the assumption that $\alpha_{\text {best }}$ or the current helicity intensity reflect the twist of an active region and that the tilt angle is related to the writhe of active regions, Canfield \& Pevtsov (1998) and Sakurai \& Hagino (2003) found a positive correlation between twist and writhe of active regions. However, Tian et al. (2001) and Lopez Fuentes et al. (2003) found a negative correlation between twist and writhe. Actually, the twist and writhe are both geometric properties of flux tubes and they can interconvert casting doubt on magnetic helicity conservation. It is reasonable to consider the relation between twist and writhe from the point of view of helicity conservation. If a twisted flux tube without initial writhe emerges from the convective zone and its writhe is caused by kink instability, the magnetic helicity and writhe should have the same sign. The accumulated relative helicity in formula (6) can be considered as the total magnetic helicity including twist and writhe. So what is the relation between the accumulated helicity and the writhe of active regions?

Note that the magnetic helicity in the corona has different sources: newly emerging magnetic flux, the footpoint motion of flux tubes and their rotation. It was shown that differential rotation cannot supply enough helicity to the corona and CMEs (Demoulin et al. 2002; Green et al. 2002; Mandrini et al. 2004). Many authors have studied the helicity of active regions. Lanbonte et al. (2007) investigated 48 X-flaring regions and 345
non-X-flaring regions. They found that a necessary condition for the occurrence of an X-flare is that the peak helicity flux exceeds a magnitude of $6 \times 10^{36} \mathrm{Mx}^{2} \mathrm{~s}^{-1}$ and that these active regions supply enough magnetic helicity for their CMEs. They also found a weak hemispheric preference of helicity injection, which might be caused by the solar differential rotation. They did not take the initial emerging phase of the active regions into consideration. Therefore, it could not reveal the character of accumulated helicity in active regions. Jeong \& Chae (2007) studied the accumulated helicity of several newly emerging active regions. They suggested that magnetic flux emergence may be the main cause of helicity injection. Tian \& Alexander (2008) investigated 19 newly emerging active regions with a similar result as Jeong \& Chae (2007). The character of accumulated helicity of active regions is, however, still unclear.

To clarify the above questions, we selected 58 active regions with simple magnetic field structures from MDI data and investigated the accumulated total helicity of the active regions by following the method of Chae et al. (2001) and the writhe of active regions as Lopez Fuentes et al. (2003) according to the evolution of the tilt angle. In Sect. 2, we present the observational data and their reduction. In Sect. 3, we describe the procedure to calculate the magnetic helicity and the tilt angle. In Sect. 4, we present the results and in Sect. 5, we give the summary and discussion.

\section{Observations and data reduction}

We use data of the full-disk line-of-sight magnetograms taken by SOHO/MDI. MDI full-disk magnetograms are recorded by a $1024 \times 1024$ CCD detector with a pixel size of $2^{\prime \prime}$. MDI magnetographs have been calibrated (Scherrer et al. 1995), and the conversion factor is $2.82 \mathrm{G} \mathrm{DN}^{-1}$ (Schrijver et al. 1997). There are two kinds of data: 1 min cadence data and 96 min cadence data. We used 96 min cadence data for this study because the noise level of this type of data is much less than that of $1 \mathrm{~min}$ data. We selected emerging active regions and calibrate them from the MDI full disk magnetographs in the period of 1996-2006.

The detailed process is the following: first, we obtained one full disk magnetogram from the Solar Active Region Monitor (Gallagher et al. 2002) every day from 1996 to 2006, since SOHO was launched. Second, we compared the magnetograms of each pair of two days. We look for new emerging active regions with existing NOAA number appearing the second day and this active region does not develop into a complex magnetic structure such as the $\gamma$ or $\delta$ types of active regions in the following several days. This active region is considered as one sample. We found 58 of such samples, 25 in the northern hemisphere and 33 in the southern hemisphere. Second, we followed this active regions until it almost arrived at the west limb. Actually, because of the MDI instrument, magnetograms which are very near to the solar limb are not appropriate for our purpose. The tracking days are between 2.3 days and 8.7 days since their emergence in our 58 samples. Thirdly, we apply nonlinear mapping, flux density interpolation and geometrical foreshortening correction to the MDI 96 min data following the method given by Chae et al. (2001). we removed the effect of differential rotation in the magnetograms with the expression

$\omega(\lambda)=a+b \sin ^{2} \lambda+c \sin ^{4} \lambda$

with $a=14.33 \mathrm{deg} / \mathrm{day}, b=-2.12 \mathrm{deg} / \mathrm{day}$ and $c=$ $-1.83 \mathrm{deg} / \mathrm{day}$ (Howard et al. 1990). The final magnetograms have new grids with a pixel size of $1^{\prime \prime}$, and the vertical field strength becomes equal to the line-of-sight strength times 

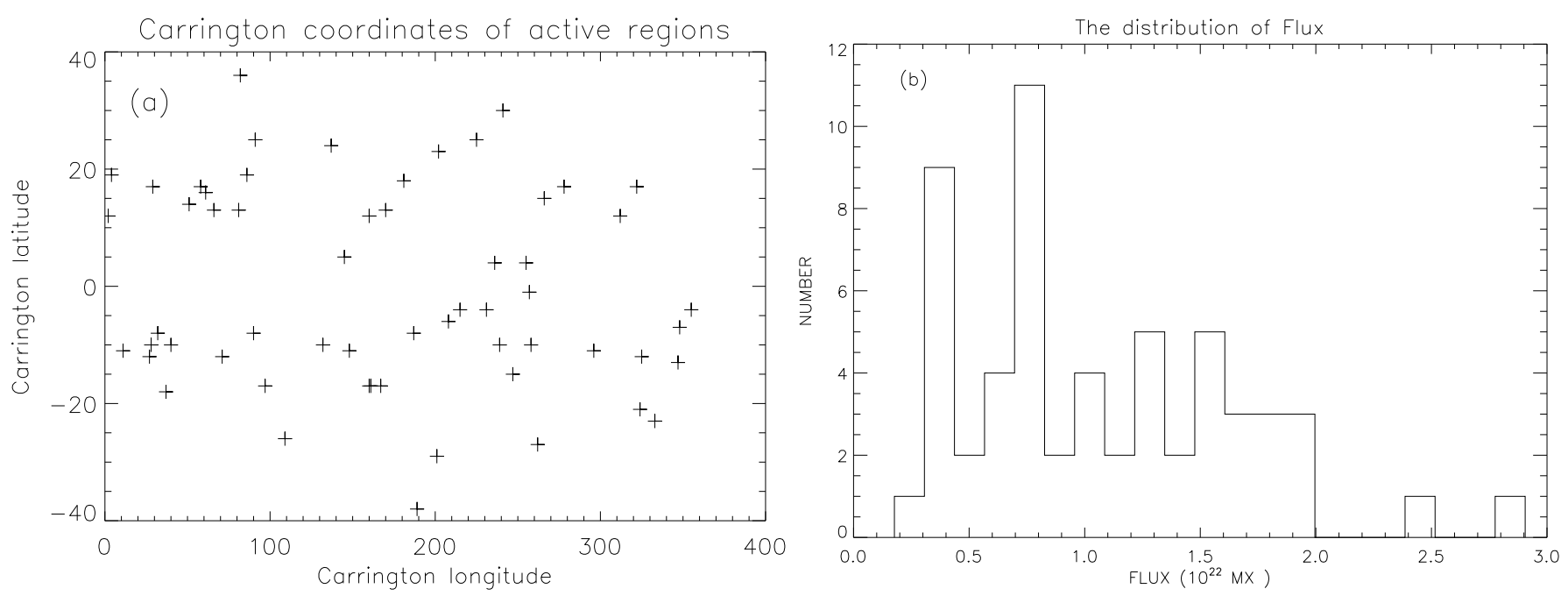

Fig. 1. Panel a) is the Carrington coordinates of the active regions and panel $\mathbf{b}$ ) is the flux distribution of the active regions.

$1 / \cos \psi$, where $\psi$ is the heliocentric angle of the region (Liu \& Zhang 2006). After this correction, the heliographic longitudes are between $-65^{\circ}$ and $65^{\circ}$ and the latitudes for all active regions are between $-40^{\circ}$ and $40^{\circ}$. Their Carrington coordinates and the unsigned flux distribution are shown in Fig. 1.

\section{Helicity and tilt angle}

\subsection{Helicity}

The magnetic helicity flux $\frac{\mathrm{d} H_{\mathrm{R}}}{\mathrm{d} t}$ can be obtained by formula (6). We use FFT to get the vector potential of magnetic field by following the method of Chae et al. (2001). We apply the LCT method to calculate the velocity of plasma in the solar photosphere by using observed line-of-sight magnetic field components. Although it is known that there are some problems using LCT (Demoulin \& Berger 2003; Schuck 2005; Welsh et al. 2004), a comparison of results obtained by LCT and other methods did not reveal a change in the main velocity pattern of photospheric plasma motion. For example, Santos et al. (2005) compared the horizontal component of the photospheric velocity obtained by using LCT, ILCT and MEF methods and found that the results are similar.

The pixel scale of the MDI data is $1.97784 \mathrm{arcsec} / \mathrm{pix}$. Physically significant transverse velocities of photospheric fluxes are less than $1.5 \mathrm{~km} \mathrm{~s}^{-1}$ (Chae et al. 2001). The pixel size of the final reduced magnetogram according to the procedure in Sect. 2 is $1^{\prime \prime}$, so the possible shift between the two reduced magnetograms is less than 12 pixels. In the LCT method, we choose the FWHM (full widths at half-maximum) of the apodizing function as $12^{\prime \prime}$. To reduce the noise effects, we set the horizontal velocity to zero in regions with a low magnetic field $(<10 \mathrm{G})$. In order to better track the emerging regions and exclude the effects from the relative quiet regions outside the emerging regions, we also set the horizontal velocity to zero in regions with low cross-correlation value $(<0.9)$. Some magnetic helicity will be lost, taken by short-lived or small-scale flows with different cross-correlation values, while the horizontal errors are localized and do not affect the final accumulated magnetic helicity within about 10\% (Chae et al. 2004; Liu \& Zhang 2006).

Pariat et al. (2005) define a new helicity flux density $G_{\theta}$, replacing that obtained by the method above. Their method provides more accurate magnetic helicity flux densities than just applying LCT. For our purpose, we only need to know the integral of the helicity flux densities, while the pattern of the helicity flux is not important for this. Note that Lim et al. (2007) compared the consistency of the use of the LCT method to measure magnetic helicity injection through the photosphere and that of the LFFF method to determine helicity. Their results support the reliability of previous method determining the injected helicity.

The accumulated magnetic helicity of an active region at a certain time is given by

$H_{\mathrm{R}}(t)=\int_{0}^{t} \frac{\mathrm{d} H_{\mathrm{R}}(t)}{\mathrm{d} t} \mathrm{~d} t$

from the time $t=0$ when the active region begins to emerge, until to a certain time $t$. The time $t=0$ is for the first magnetogram and the time $t$ is for the last one. As the helicity helicity flux is obtained in a discrete form, we utilize a summation given by

$H_{\mathrm{R}}\left(t_{n}\right)=\sum_{t=0}^{n} \frac{\mathrm{d} H_{\mathrm{R}}\left(t_{i}\right)}{\mathrm{d} t} \cdot \Delta t_{i}$

to obtain accumulated helicity in this paper.

\subsection{Tilt angle}

The leading polarity of the $23 \mathrm{rd}$ solar cycle is positive (negative) in the northern (southern) hemisphere. We define the original point at the barycenter of the positive (negative) polarity on the northern (southern) hemisphere. The $x$ direction is the solar rotation direction. The $y$ direction is from south to north. The angle $\left(0^{\circ}<\mathrm{Ta} \leq 360^{\circ}\right)$ of the vector from the original point to the barycenter of the following polarity of the active region is defined as the tilt angle, described in Fig. 2. To reduce the noise of the calculated tilt angle, only magnetic field strengths exceeding $15 \mathrm{G}$ are considered for the calculation of the tilt angle of an active region.

In our analysis, we removed the influence of differential rotation as described by Eq. (7) before calculating the magnetic helicity and tilt angle. Let us consider an active region extended $10^{\circ}$ in the south-north direction and with a latitude of $0^{\circ} \sim 30^{\circ}$, The rotation rate is between $0.0656^{\circ} /$ day and $0.544^{\circ}$ day. For a typical emerging active region in our samples, the AR will take 
Northern hemisphere

Southern hemisphere
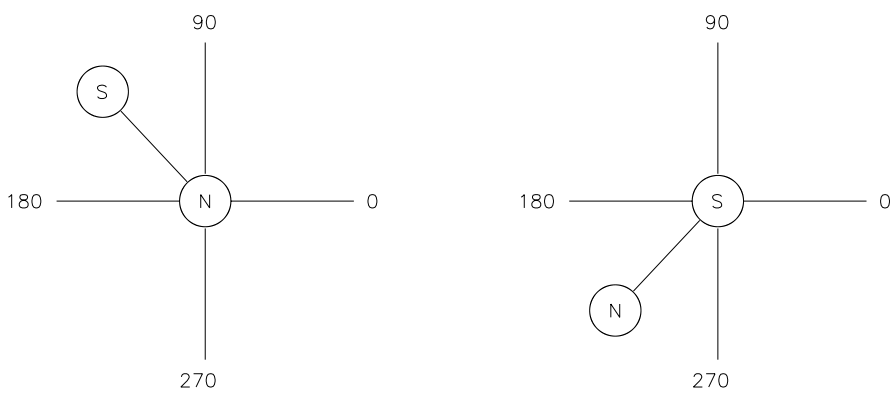

Fig. 2. Tilt angle (Ta) in this paper. The left (right) one is for the northern (southern) hemisphere. Ta is between $0^{\circ} \sim 360^{\circ}$.

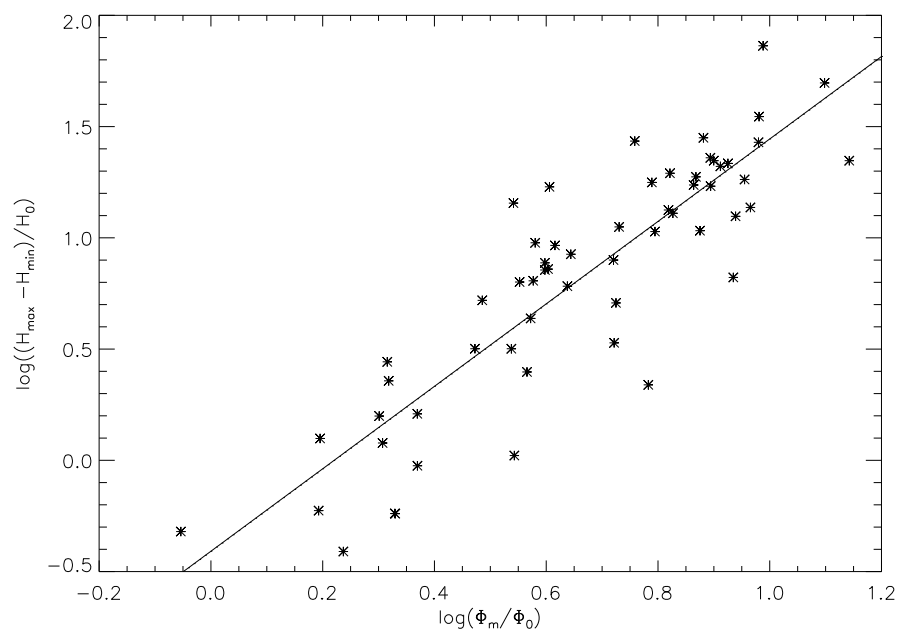

Fig. 3. Integrated helicity flux as a function of total AR magnetic flux $\left(H_{0}=10^{41} M X^{2}, \Phi_{0}=10^{21} M X\right)$.

about ten days to pass through the solar disk. The maximum correction for the tilt angle would be 5.44 .

\section{Results}

\subsection{Magnetic flux and helicity}

Figure 3 depicts integrated magnetic helicity fluxes $\left(H_{\max }-H_{\min }\right)$ vs. the total magnetic flux of the ARs. The magnetic flux $\Phi_{\mathrm{m}}$ is one-half of the maximum sum of the unsigned positive and negative magnetic fluxes. The best linear fit line (solid line in Fig. 3) shows the relation between integrated magnetic helicity fluxes and magnetic fluxes

$\log \frac{H_{\max }-H_{\min }}{H_{0}}=a \log \frac{\Phi_{\mathrm{m}}}{\Phi_{0}}+b$

where $a=1.85, b=-0.41, H_{0}=10^{41} M X^{2}$ and $\Phi_{0}=10^{21} M X$. This indicates that the accumulated magnetic helicity is proportional to the exponent of magnetic flux $\left(|H| \propto \Phi^{1.85}\right)$. Jeong $\&$ Chae (2007) found an exponent of 1.3 for the coefficient " $a$ " from four active regions. Labonte et al. (2007) found a similar value of 1.8 for " $a$ ". If we suppose that the coronal part of each AR is represented by a single semicircular loop, the average twist $\mathrm{Tw}$ in these loops would be $10^{b} H_{0} / \Phi_{0}^{2}=$ 0.039 turns. Nindos et al. (2003) found values between 0.01 and 0.17. Labonte et al. (2007) found a value of 0.022 .
Tian \& Alexander (2008) found a similar value, 0.03. For a typical coronal loop with diameter $d=100 \mathrm{Mm}$ at the photosphere foot-points, the twist rate $q$ (radians per unit length) would be Tw $/(\pi d / 2)=2.48 \times 10^{-12} \mathrm{~cm}^{-1}$. This result is similar to that in the survey of Labonte et al. (2007), who obtained $1.4 \times 10^{-12} \mathrm{~cm}^{-1}$ for $48 \mathrm{X}$-flaring regions and 345 non-X-flaring regions. However, these results are all one order smaller than the average twist rates of $10^{-11} \mathrm{~cm}^{-1}$ in AR loops by using vector magnetograms to find $\alpha_{\text {best }}$ (Pevtsov et al. 1995). Note that the $\alpha_{\text {best }}$ is related to the twist rate $q$ by $\alpha_{\text {best }}=2 q$ for the thin flux rope model (Longcope et al. 1998) and an debate that whether such an assumption is valid for one active region still exist (e.g. Leka et al. 2005).

\subsection{Two typical solar active regions}

In order to better understand the accumulation of magnetic helicity and tilt angle evolution in newly emerging ARs, we have divided our samples into two groups $\mathrm{A}$ and $\mathrm{B}$, according to the resulting relation between accumulated magnetic helicity and the rotation of the tilt angles. We neglect the evolution detail of magnetic helicity and tilt angle. We focus on the final accumulated helicity $H(t)$ and the change of tilt angle $\Delta \mathrm{Ta}=\mathrm{Ta}(t)-\mathrm{Ta}(0)$. In the following, we describe the two groups according to the relation between $H(t)$ and $\Delta$ Ta. One example will be given for each type of group.

Group A: $H \cdot \Delta \mathrm{Ta}>0$. The accumulated helicity of ARs is negative (positive) when the tilt angle rotates clockwise (counter-clockwise). For example, AR 9931 emerged on April 30, 2002 and disappeared near the west limb on May 9, 2002. The evolution of the line-of-sight magnetic field and the time profile of accumulated magnetic helicity and tilt angle are shown in Fig. 4.

Group B: $H \cdot \Delta \mathrm{Ta}<0$. The accumulated helicity of ARs is positive (negative) when the tilt angle rotates clockwise (counter-clockwise). AR10481, a sample of this type group, emerged from the solar surface on Oct. 16, 2003 and disappeared near the west limb on Oct. 21,2003 . The evolution of the line-of-sight magnetic field and the time profile of accumulated magnetic helicity and tilt angle are shown in Fig. 5.

There are 43 ARs $(74 \%)$ of the samples in Group A. 19 ARs are in the northern hemisphere and 24 ones are in the southern hemisphere. 15 ARs $(26 \%)$ of the samples are in group B. 6 ARs are in the northern hemisphere and 9 are in the southern hemisphere. The detailed values are listed in Table A.1 (Table A.2) of Appendix A.

All active regions in our samples obey the Hale-Nicholson law (Hale \& Nicholson 1925): the leading polarities are always positive (negative) in the northern (southern) hemisphere in the 23rd solar cycle. Thus one active region will satisfy Joy's law when the leading polarities are closer to the equator than the trailing polarities (Hale 1919) if its final tilt angle when we stop to follow the $\mathrm{AR}$ is $90^{\circ}<\mathrm{Ta}(t)<180^{\circ}\left(180^{\circ}<\mathrm{Ta}(t)<270^{\circ}\right)$ in the northern (southern) hemisphere according to the definition of tilt angle in our study. In the southern hemisphere, among the 22 ARs which follow the Joy's law, 17 (77\%) belongs to group A while among the 11 ARs which do not follow Joy's law, 7 (63\%) belong to the group A. In the northern hemisphere, among the 14 ARs which follow Joy's law, 11 (79\%) belong to the group A while among the 11 ARs which do not follow Joy's law, 8 (73\%) belong to the group A. The probability is $78 \%$ for an AR 

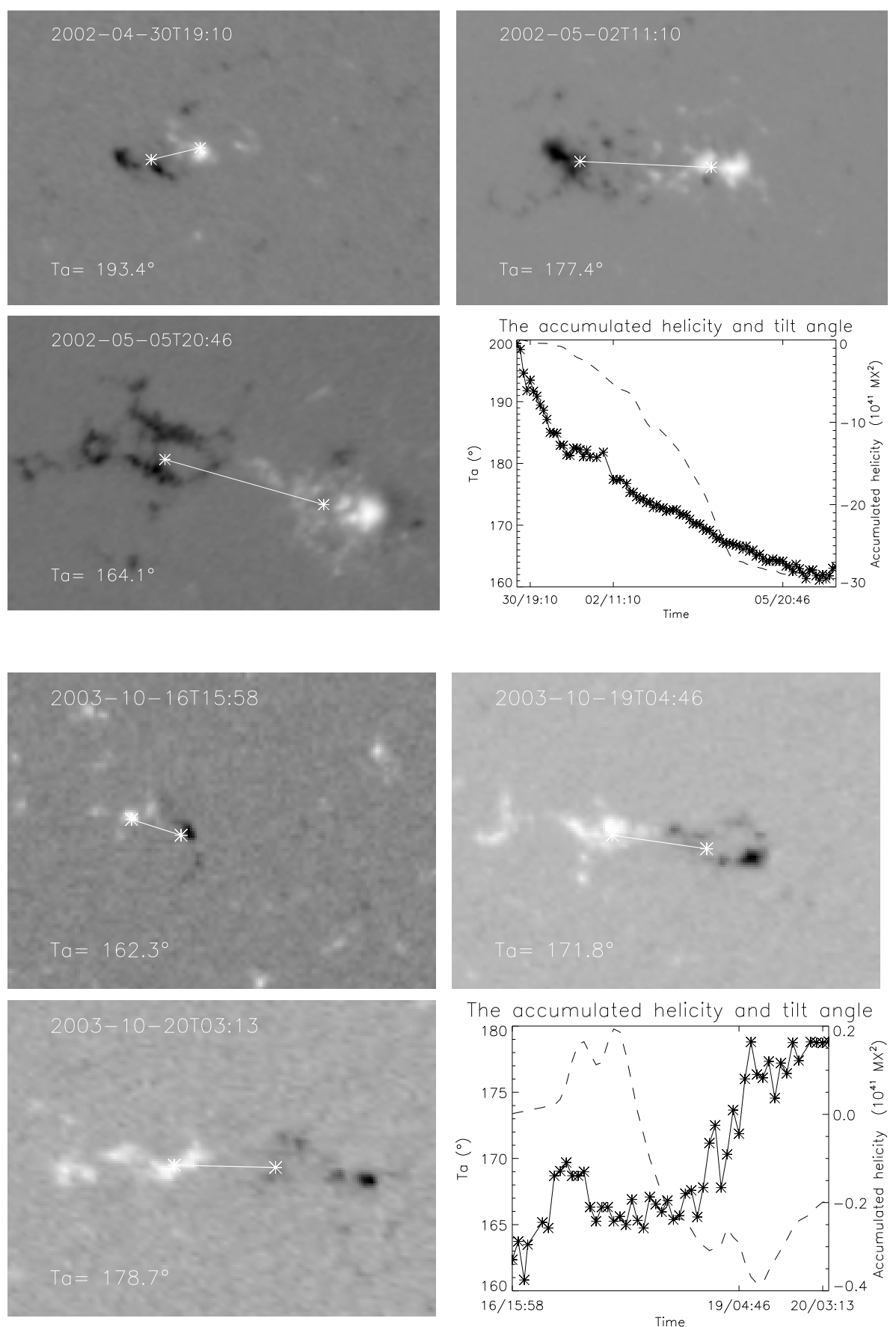

Fig. 4. AR09931: an example of active regions belonging to the group $\mathrm{A}$. The top and bottom left images reflect the evolution of this active region at three different moments of time. The tilt angle (Ta) is given. The bottom right plot shows the temporary evolution of accumulated magnetic helicity and tilt angle. The left ordinate of this plot corresponds to the tilt angle (asterisk) and the right is accumulated helicity (dashed line).

Fig. 5. AR10481: an example of active regions belonging to the group $\mathrm{B}$. The top and bottom left images reflect the evolution of this active region at three different moments of time. The tilt angle (Ta) is given. The bottom right plot shows the temporary evolution of accumulated magnetic helicity and tilt angle. The left ordinate of this plot corresponds to the tilt angle (asterisk)and the right is accumulated helicity (dashed line). to be in group A if it follows Joy's law, which is higher than the probability of $68 \%$ if it does not follow Joy's law. The details of the distribution of the two groups according to Joy's law are listed in Table 1.

Structural chirality and magnetic helicity of solar active regions tend to show negative (positive) helicity in the northern (southern) hemisphere according to the study of current helicity and $\alpha_{\text {best }}$ (Seehafer 1990; Bao \& Zhang 1998). In the southern hemisphere, $19(57.6 \%)$ of 33 ARs with positive helicity satisfy the hemisphere rule. Among the 19 ARs which satisfy the hemisphere rule, $14(74.0 \%)$ belong to group A while among the 14 ARs which do not follow the hemisphere rule, 10 (71\%) belong to group A. In the northern hemisphere, 14 ARs $(56.0 \%)$ of 25 have negative helicity and satisfy the hemisphere rule.
Table 1. Distribution of the two groups according to the Joy's law.

\begin{tabular}{llllll}
\hline \hline & Joy $^{a}$ & Joy & Non-Joy $^{b}$ & Non-Joy & \\
Hemisphere $^{c}$ & $\mathrm{~S}$ & $\mathrm{~N}$ & $\mathrm{~S}$ & $\mathrm{~N}$ & Total \\
\hline $\mathrm{A}$ & $17(77 \%)$ & $11(79 \%)$ & $7(63 \%)$ & $8(73 \%)$ & $43(74 \%)$ \\
$\mathrm{B}$ & $5(23 \%)$ & $3(21 \%)$ & $4(37 \%)$ & $3(27 \%)$ & $15(26 \%)$ \\
Total & 22 & 14 & 11 & 11 & $58(100 \%)$ \\
\hline
\end{tabular}

$a$ "Joy" means that active regions satisfy Joy's law. "Non-Joy" means that active regions disobey Joy's law. ${ }^{c} \mathrm{~N}$ (S) stands for the northern (southern) hemisphere.

Among the 14 ARs which satisfy the hemisphere rule, $13(93 \%)$ belong to group A while among the 11 ARs which do not follow 
Table 2. Distribution of the two groups according to the hemisphere helicity rule.

\begin{tabular}{llllll}
\hline \hline & $\mathrm{HHR}^{a}$ & $\mathrm{HHR}$ & \multicolumn{2}{l}{ Non-HHR $^{b}$} & Non-HHR \\
Hemisphere $^{c}$ & $\mathrm{~S}$ & $\mathrm{~N}$ & $\mathrm{~S}$ & $\mathrm{~N}$ & Total \\
\hline $\mathrm{A}$ & $14(74 \%)$ & $13(93 \%)$ & $10(71 \%)$ & $6(55 \%)$ & $43(74 \%)$ \\
$\mathrm{B}$ & $5(26 \%)$ & $1(7 \%)$ & $4(29 \%)$ & $5(45 \%)$ & $15(26 \%)$ \\
Total & 19 & 14 & 14 & 11 & $58(100 \%)$ \\
\hline
\end{tabular}

${ }^{a}$ HHR means that the active regions satisfy hemisphere helicity rule. ${ }^{b}$ Non-HHR means that the active regions disobey hemisphere helicity rule. ${ }^{c} \mathrm{~N}(\mathrm{~S})$ stands for the northern (southern) hemisphere.

the hemisphere rule, 6 (55\%) belong to group A. The probability is $82 \%$ for an AR to be in the group A if given that it follows the hemisphere rule, which is higher than the probability of $64 \%$ if it does not follow the hemisphere rule. The details of the distribution of the two groups according to the hemisphere rule are given in Table 2.

\section{Summary and discussion}

We have investigated the accumulation of magnetic helicity in 58 newly emerging simple bipolar solar active regions. We found that $74 \%$ of these ARs belong to group A, with negative (positive) helicity when the connecting line of the weighting centers of opposite polarities in the ARs rotates clockwise (counter-clockwise) $(\mathrm{H} \cdot \Delta \mathrm{Ta}>0)$. Assuming that a clockwise rotation of the polarities indicates a negative (right-handed) writhe, and counterclockwise represents a positive (left-handed) writhe (Lopez et al. 2003), it is found that the majority of ARs in newly emerging ARs will have a writhe $(W)$ that has the same sign as the accumulated magnetic helicity $H(H \cdot W>0)$.

There are two possible sources for the writhe of the emerging magnetic flux tubes: a kink instability and the Coriolis force. Let us suppose a flux tube emerging from the convection zone with a $\Omega$ planar shape and that there is only a twist without an initial writhe in this flux tube. The initial magnetic helicity can be either $H>0, H<0$ or $H=0$. A certain amount of twist is needed to prevent the flux tube from being destroyed by the hydrodynamic vortex behind it during its emergence in the convection zone (Fan et al. 1998). Simulations of flux tube emergence (Schüssler et al. 1979; Moreno-Insertis \& Emonet 1996; Emonet \& Moreno-Insertis 1998; Fan et al. 2003; Cheung et al. 2006) also showed that untwisted flux tubes are very unlikely to emerge. So we consider the two situations $H>0$ and $H<0$.

When this flux tube emerges through the convective zone and only the Coriolis force deforms its main axis to introduce writhe helicity, the acquired writhe will be positive (negative) in the northern (southern) hemisphere. In our sample, 15 (45\%) of 33 have a negative writhe in the southern hemisphere, and 7 $(28 \%)$ of 25 have a positive writhe in the northern hemisphere. A considerable number of ARs have opposite writhe. Hence, the Coriolis force is not the only source of the writhe of emerging flux tube.

When a horizontal flux tube emerges and the acquired writhe for this flux tube results from kink instability, the helicity of the flux tube will have the same sign as its writhe $(H \cdot W>0)$ due to conservation of helicity. This would lead to a clockwise (counter-clockwise) rotation of the apex portion of the rising tube when viewed from the top, if this kinked flux tube carries negative (postive) helicity (Fan et al. 1999). In our study, 43 (74\%) active regions in group A satisfy this condition $(H \cdot W>$ 0 ). Moreover, the probability is $82 \%$ for an AR to be in group A if it follows the hemisphere helicity rule, which is more than the probability $64 \%$ if it does not follow the hemisphere rule. The rotation of the apex portion of the kinked flux tubes induced by the Coriolis force is hemisphere-dependent and is clockwise (counterclockwise) in the northern (southern) hemisphere. Hence, the Coriolis force will enhance (work against) the trend of clockwise (counter-clockwise) rotation of a kinked negative (positive) twisted tube in the northern hemisphere and will enhance (work against) the trend of counter-clockwise (clockwise) rotation of a kinked positive (negative) twisted tube in the southern hemisphere (Fan et al. 1999). So one AR satisfying the hemisphere helicity rule has a higher probability of belonging to group A than the AR disobeying the hemisphere helicity rule. The kink instability could well explain the relation between the magnetic helicity and tilt angle evolution for the emerging active regions in our study.

This result is different to Tian et al. (2001) and Lopez et al. (2003). Tian et al. (2001) found a negative relation between twist and writhe for the 286 active regions. They concluded that the twist is caused by the Coriolis force distorting the apex of emerging $\Omega$-loops, which introduces both twist and writhe in originally untwisted magnetic fields. Lopez et al. (2003) studied the evolution of 22 ARs and found evidence of kinking in about $35 \%$ of these regions while $41 \%$ of these regions showed a twist-tilt relationship indicative of having resulted from the Coriolis force. The kink instability is not an obvious outcome of their papers. Holder et al. (2004) found that those regions that closely follow Joy's law do not show any twist-tilt dependence and regions that strongly depart from Joy's law show a significantly larger average twist and very strong twist-tilt dependence. Those authors suggested that the active regions strongly departing from Joy's law come from kinking of flux tubes. Our study also supports the existence of kink instability in the emerging flux tube.

What has caused the difference? One important reason is that we analyzed the emerging phase of the ARs ( 2 to 9 days after emergence). In the previous investigation, the authors considered this relation over a longer period of time. Lopez et al. (2000, 2003) selected samples from several solar Carrington rotations. Most active regions in Tian et al. (2001) are also not newly emerging, young ones. Holder et al. (2004) pointed that the Tian et al. (2001) analysis did not correct for the mutual latitude dependence between twist and tilt and that any signature of kink instability would have been suppressed in their data set. Tian et al. (2005) investigated $\delta$-sunspots and they found a positive relation between twist and writhe, showing evidence of kink instability. The emerging flux tubes may interact with large-scale vortical motions of the plasma in the convection zone, as pointed out by Lopez et al. (2000, 2003), which would suppress any signature of kink instability.

$56.0 \%(57.6 \%)$ of ARs in the northern (southern) hemisphere supply negative (positive) helicity to the upper photosphere. This result is similar to that of the survey of magnetic helicity injection carried out by Labonte et al. (2007), 57\% (60\%) of ARs have negative (positive) magnetic helicity flux in the northern (southern) hemisphere. They conjectured that the weak hemispheric preference of helicity injection, positive in the south and negative in the north, is caused by the differential rotation at the solar surface. Helicity injection in which the effect of differential rotation has been removed tends to have the opposite sign. In our analysis, we have removed the differential rotation at the solar surface. The strong magnetic flux emergence is the most 
important source of coronal helicity and the injection of magnetic helicity by differential rotation is a factor 2 to 10 lower than the photospheric helicity rates computed from AR internal motion (Demoulin \& Pariat 2009). So our result would exhibit the same trend if did not remove the differential rotation. Note that all of the above trends are weaker than most of those based on the $\alpha_{\text {best }}$, current helicity and shapes of sigmoidal coronal loops. In those studies, there is a 50\%-79\% (57.5\%-78\%) negative (positive) helicity preference in the northern (southern) hemisphere. Our method is more directly determining the accumulated magnetic helicity than the previous methods, which may cause the weaker helicity preference.

Acknowledgements. We appreciate the referee's careful reading of the manuscript and many constructive comments, which greatly improved the paper. We are also grateful to the Huairou Solar Observing Station and SOHO/MDI group. This study is supported by grants 10673016 of National Natural Science Foundation of China, 2006CB806301 of the National Basic Research Program of China and a sandwich-Ph.D. grant of the Max-Planck Society.

\section{Appendix A: All results for the northern and southern hemisphere}

Table A.1. Distribution of accumulated helicity and tilt angle evolution in the northern hemisphere.

\begin{tabular}{|c|c|c|c|c|c|c|}
\hline Type & NOAA & Helicity sign ${ }^{a}$ & Helicity $^{b}$ & Rotation $^{c}$ & $\Delta \mathrm{Ta}^{d}$ & Joy's law ${ }^{e}$ \\
\hline \multirow{19}{*}{ A } & 8164 & - & -7.85 & - & -55.8 & $\mathrm{~F}$ \\
\hline & 10045 & - & -20.5 & - & -45.8 & $\mathrm{~F}$ \\
\hline & 10227 & - & -10.3 & - & -25 & $\mathrm{~F}$ \\
\hline & 10232 & - & -0.383 & - & -22.5 & $\mathrm{~F}$ \\
\hline & 8117 & - & -0.49 & - & -9.58 & $\mathrm{~T}$ \\
\hline & 8843 & - & -1.47 & - & -45.9 & $\mathrm{~T}$ \\
\hline & 9728 & - & -3.37 & - & -37.7 & $\mathrm{~T}$ \\
\hline & 9931 & - & -27.5 & - & -36.1 & $\mathrm{~T}$ \\
\hline & 10268 & - & -13.8 & - & -33 & $\mathrm{~T}$ \\
\hline & 10385 & - & -3.84 & - & -10.0 & $\mathrm{~T}$ \\
\hline & 10461 & - & -6.87 & - & -18.3 & $\mathrm{~T}$ \\
\hline & 10838 & - & -4.24 & - & -13.57 & $\mathrm{~T}$ \\
\hline & 10879 & - & -4.75 & - & -55.1 & $\mathrm{~T}$ \\
\hline & 8116 & + & 8.69 & + & 36.9 & $\mathrm{~F}$ \\
\hline & 9495 & + & 6.17 & + & 7.93 & $\mathrm{~F}$ \\
\hline & 10132 & + & 73.0 & + & 35 & $\mathrm{~F}$ \\
\hline & 10839 & + & 1.42 & + & 37.1 & $\mathrm{~F}$ \\
\hline & 9144 & + & 49.5 & + & 67.2 & $\mathrm{~T}$ \\
\hline & 10480 & + & 0.16 & + & 12.5 & $\mathrm{~T}$ \\
\hline \multirow{6}{*}{ B } & 10646 & - & -15.0 & + & 66.9 & $\mathrm{~F}$ \\
\hline & 8052 & + & 9.93 & - & -15.0 & $\mathrm{~F}$ \\
\hline & 10214 & + & 12.3 & - & -20.7 & $\mathrm{~F}$ \\
\hline & 8722 & + & 2.73 & - & -25.0 & $\mathrm{~T}$ \\
\hline & 8123 & + & 16.0 & - & -32.5 & $\mathrm{~T}$ \\
\hline & 9063 & + & 24.5 & - & -0.8 & $\mathrm{~T}$ \\
\hline
\end{tabular}

${ }^{a}$ The sign $+(-)$ in the helicity column represents positive (negative) helicity. ${ }^{b}$ The unit of helicity is $10^{41} M x^{2}{ }^{c}{ }^{c}$ The sign $-(+)$ in the rotation direction column represents the active region rotating clockwise (counter-clockwise). ${ }^{d}$ The unit of $\Delta T a$ is degree. ${ }^{e} \mathrm{~T}(\mathrm{~F})$ shows that the active region satisfies (disobeys) Joy's law.
Table A.2. Distribution of accumulated helicity and tilt angle evolution in the southern hemisphere.

\begin{tabular}{|c|c|c|c|c|c|c|}
\hline Type & NOAA & Helicity sign $^{a}$ & Helicity $^{\mathrm{b}}$ & Rotation $^{\mathrm{c}}$ & $\Delta \mathrm{Ta}^{\mathrm{d}}$ & Joy's law \\
\hline \multirow{24}{*}{ A } & 9139 & - & -20.5 & - & -22.8 & $\mathrm{~F}$ \\
\hline & 9399 & - & -14.1 & - & -18.4 & $\mathrm{~F}$ \\
\hline & 10569 & - & -5.93 & - & -14.3 & $\mathrm{~F}$ \\
\hline & 9897 & - & -1.68 & - & -37.9 & $\mathrm{~F}$ \\
\hline & 10078 & - & -0.021 & - & -27.1 & $\mathrm{~F}$ \\
\hline & 9873 & - & -26.8 & - & -61 & $\mathrm{~T}$ \\
\hline & 8118 & - & -6.39 & - & -30.2 & $\mathrm{~T}$ \\
\hline & 10141 & - & -5.91 & - & -70.3 & $\mathrm{~T}$ \\
\hline & 10692 & - & -4.12 & - & -22.4 & $\mathrm{~T}$ \\
\hline & 10135 & - & -0.13 & - & -31.3 & $\mathrm{~T}$ \\
\hline & 10298 & + & 2.24 & + & 11 & F \\
\hline & 10671 & + & 21.6 & + & 36.9 & F \\
\hline & 10270 & + & 1.01 & + & 53.4 & $\mathrm{~T}$ \\
\hline & 10426 & + & 1.07 & + & 0.2 & $\mathrm{~T}$ \\
\hline & 10072 & + & 1.68 & + & 16.6 & $\mathrm{~T}$ \\
\hline & 10291 & + & 2.12 & + & 25.5 & $\mathrm{~T}$ \\
\hline & 10376 & + & 7.08 & + & 21.9 & $\mathrm{~T}$ \\
\hline & 8016 & + & 8.43 & + & 60.4 & $\mathrm{~T}$ \\
\hline & 10828 & + & 9.02 & + & 39 & $\mathrm{~T}$ \\
\hline & 10869 & + & 17.2 & + & 15.8 & $\mathrm{~T}$ \\
\hline & 9710 & + & 15.9 & + & 37.9 & $\mathrm{~T}$ \\
\hline & 10591 & + & 17.7 & + & 28 & $\mathrm{~T}$ \\
\hline & 9396 & + & 22.2 & + & 8.58 & $\mathrm{~T}$ \\
\hline & 9417 & + & 31.9 & + & 8.11 & $\mathrm{~T}$ \\
\hline \multirow{9}{*}{ B } & 10684 & - & -0.11 & + & 4.1 & $F$ \\
\hline & 10481 & - & -0.2 & + & 16.5 & $\mathrm{~F}$ \\
\hline & 10454 & - & -2.5 & + & 10.5 & $\mathrm{~T}$ \\
\hline & 8167 & - & -1.28 & + & 2.9 & $\mathrm{~T}$ \\
\hline & 10006 & + & 0.351 & - & -65.3 & $\mathrm{~F}$ \\
\hline & 10489 & + & 1.14 & - & -18.7 & $\mathrm{~F}$ \\
\hline & 8174 & + & 1.49 & - & -6.3 & $\mathrm{~T}$ \\
\hline & 10499 & + & 3.15 & - & -6.9 & $\mathrm{~T}$ \\
\hline & 10837 & + & 3.6 & - & -28 & $\mathrm{~T}$ \\
\hline
\end{tabular}

${ }^{a}$ The sign $+(-)$ in the helicity column represents positive (negative) helicity. ${ }^{b}$ The unit of helicity is $10^{41} M x^{2} .{ }^{c}$ The sign $-(+)$ in the rotation direction column represents the active region rotating clockwise (counter-clockwise). ${ }^{d}$ The unit of $\Delta \mathrm{Ta}$ is degree. ${ }^{e} \mathrm{~T}(\mathrm{~F})$ shows that the active region satisfies (disobeys) Joy's law.

\section{References}

Abramenko, V. I., Wang, T. J., \& Yurchishin, V. B. 1996, Sol. Phys., 168, 75 Bao, S. D., \& Zhang, H. Q. 1998, ApJ, 496, L43

Berger, M. A. 1999, Plasma Phys. Control. Fusion, 41, B167 Berger, M. A., \& Field, G. B. 1984, J. Fluid Mech., 147, 133

Canfield, R. C., \& Pevtsov, A. A. 1998, Sol. Phys., 182, 145

Chae, J., Wang, H., Qiu, J., et al. 2001, ApJ, 560, 476

Cheung, M. C. M., Schüssler, M., \& Moreno-Insertis, F. 2006, ASPC, 354, 97

Démoulin, P., \& Pariat, E. 2009, Adv. Space Res., 43, 1013

Démoulin, P., \& Berger, M. A. 2003, Sol. Phys., 215, 203

Démoulin, P., Mandrini, C. H., van Driel-Gesztelyi, L., Lopez Fuentes, M. C., \&

Aulanier, G. 2002, Sol. Phys., 207, 87

Emonet, T., \& Moreno-Insertis, F. 1998, ApJ, 492, 804

Fan, Y., Zweibel, E. G., \& Lantz, S. R. 1998, ApJ, 493, 480

Fan, Y., Zweibel, E. G., Linton, M. G., \& Fisher, G. H. 1999, ApJ, 521, 460

Fan, Y., Abbett, W. P., \& Fisher, G. H. 2003, ApJ, 582, 1206

Fisher, G. H., Fan, Y., Longcope, D. W., Linton, M. G., \& Pevtsov, A. A. 2000, Sol. Phys., 192, 119 
Freeland, S. L., \& Handy, B. N. 1998, Sol. Phys., 182, 497

Gallagher, P. T., Moon, Y.-J., \& Wang, H. 2002, Sol. Phys., 209, 171

Green, L. M., Lopez Fuentes, M. C., Mandrini, C. H., et al. 2002, Sol. Phys., 208, 43

Hale, G. E., \& Nicholson, S. B. 1925, ApJ, 62, 270

Hale, G. E., Ellerman, F., Nicholson, S. B., \& Joy, A. H. 1919, ApJ, 49, 153

Holder, Z. A., Canfield, R. C., McMullen, R. A., et al. 2004, ApJ, 611, 1149

Howard, R. F., Harvey, J. W., \& Forgach, S. 1990, Sol. Phys., 130, 295

Jeong, H., \& Chae, J. 2007, ApJ, 671, 1022

Kuzanyan, K. M., Lamburt, V. G., Zhang, H., \& Bao, S. 2003, Chinese J. Astron. Astrophys., 3, 257

LaBonte, B. J., Georgoulis, M. K., \& Rust, D. M. 2007, ApJ, 671, 955

Leka, K. D., Fan, Y., \& Barnes, G. 2005, ApJ, 626, 1091L

Lim, E., Jeong, H., \& Chae, J. 2007, ApJ, 656, 1167

Liu, J., \& Zhang, H. 2006, Sol. Phys., 234, 21

Lopez Fuentes, M. C., Demoulin, P., Mandrini, C. H., \& Driel-Gesztelyi, L. V. 2000, ApJ, 544, 540

Lopez Fuentes, M. C., Demoulin, P., Mandrini, C. H., \& Pevtsov, A. A. 2003, A\&A, 397, 305

Mandrini, C. H., Demoulin, P., van Driel-Gesztelyi, L., Green, L. M., \& Lopez Fuentes, M. C. 2004, Ap\&SS, 290, 319
Moreno-Insertis, F., \& Emonet T. 1996, ApJ, 472, L53

Pariat, E., Demoulin, P., \& Berger, M. A. 2005, A\&A, 439, 1191

Pevtsov, A., Canfield, R., \& Metcalf, T. 1995, ApJ, 440, L109

Sakurai, T., \& Hagino, M. 2003, J. Korean A. S., 36, 7

Santos, J. C. , Buechner, J., Alves, M. V., Nikutowski, B., \& Zhang, H. 2005, ESASP, 596E, 63S

Scherrer, P. H., Bogart, R. S., Bush, R. I., et al. 1995, Sol. Phys., 162, 129

Schrijver, C. J., Title, A. M., van Ballegooijen, A. A., Hagenaar, H. J., \& Shine, R. A. 1997, ApJ, 487, 424

Schuck, P. W. 2005, ApJ, 632, L53

Schüssler, M. 1979, A\&A, 71, 79

Seehafer, N., Gellert, M., Kuzanyan, K. M., \& Pipin, V. V. 2003, Adv. Space Res., 32, 1819

Taylor, J. B. 1974, Phys. Rev. Lett., 33, 1139

Tian, L., \& Alexander, D. 2008, ApJ, 673, 532

Tian, L., Alexander, D., Liu, Y., \& Yang, J. 2005, Sol. Phys., 229, 63

Tian, L. R., Bao, S. D., Zhang, H. Q., \& Wang, H. N. 2001, A\&A, 374, 294

Welsch, B. T., Fisher, G. H., Abbett, W. P., \& Regnier, S. 2004, ApJ, 610, 1148

Woltjer, L. 1958, Proc. Natl Acad. Sci. USA, 44, 480

Zhang, H. 2006, Ap\&SS, 305, 211

Zhang, M., Flyer, M., \& Low, B. 2006, ApJ, 644, 575 